\title{
Feedback Control and the Arrow of Time
}

\author{
Tryphon T. Georgiou and Malcolm C. Smith
}

\begin{abstract}
The purpose of this paper is to highlight the central role that the time asymmetry of stability plays in feedback control. We show that this provides a new perspective on the use of doubly-infinite or semi-infinite time axes for signal spaces in control theory. We then focus on the implication of this time asymmetry in modeling uncertainty, regulation and robust control. We point out that modeling uncertainty and the ease of control depend critically on the direction of time. We also discuss the relationship of this control-based time-arrow with the well known arrows of time in physics.
\end{abstract}

\section{INTRODUCTION}

The origin and implications of the "arrow of time" is one of the deepest and least understood subjects of physics. The "arrow" is an intrinsic part of the world as we know it. Yet its emergence in thermodynamics and cosmology, from physical laws which are apparently impervious to it, remains a controversial subject [29]. At first sight, this subject may seem unconnected with the theory of feedback control. However, starting from the very basic fact that our notion of stability in the sense of Lyapunov is time-asymmetric, we argue that the "arrow of time" does have important implications on modeling and uncertainty, robustness of stability, as well as on the topology for the study of the dynamics of feedback interconnections.

The circle of ideas that gave rise to this paper began in a short note published by the authors thirteen years ago [8]. There, it was pointed out that the doubly-infinite time axis presents some "intrinsic difficulties" for developing a suitable input-output systems theorydifficulties that are not present in the semi-infinite time axis setting. These difficulties are not mere mathematical technicalities. Rather, they relate fundamentally to the consistency of the theory of stabilizability across different frameworks. Subsequently, a number of papers were written which shed light on the problem [22], [23], [24], [15], [16], [17]. The present paper takes a fresh look and traces the origin of the "puzzle" to the arrow of Lyapunov stability, and then, explores the relevance of this arrow to the topology of dynamical systems and feedback theory.

The relationship of the modern theory of dynamical systems with classical physics and thermodynamics is a developing one. A classical contribution by Nyquist and Johnson [28], [18] is a derivation of the electromotive force due to thermal agitation in conductors. In [4] the issue of irreversibility is treated from the point of view of stochastic control theory. More

This work was partially supported by the National Science Foundation. T.T. Georgiou is with Department of Electrical and Computer Engineering, University of Minnesota, Minneapolis, MN 55455; tryphon@ece.umn.edu. M.C. Smith is with the Department of Engineering, University of Cambridge, Cambridge, CB2 1PZ, U.K.; mcs@eng.cam.ac.uk 
recently [13] has sought to formalize classical thermodynamics in the mathematical language of modern dynamical systems (see also [5]). In [27] information flow and entropy have been studied in the context of the Kalman filter. In [31] it is shown that a linear macroscopic dissipative system can be approximated by a linear lossless microscopic system over arbitrary long time intervals. Our point of view here is influenced by [29] and is somewhat different to the above references in that our main goal is to highlight a time-asymmetry, point out its implications, and discuss its relationship to other well-known asymmetries.

The present paper begins by providing a new explanation of the issues raised in [8] with regard to an input-output theory for the doubly-infinite time axis. In Section III we introduce the time-conjugation operator and discuss the implications of the time-arrow in optimal control problems. In Section IV we analyse the effect of the time-arrow on modelling uncertainty; we show that dynamical systems which are close in the usual sense, that a common controller can stabilise and give similar closed-loop responses for either, may not be close when the time-arrow is reversed. Then, in Section $\mathrm{V}$, we further illuminate the inherent time-asymmetry in our ability to control a dynamical system with two specific examples. These can be thought of as examples of time irreversible feedback phenomena (see Section $\mathrm{V}-\mathrm{B}$ ). In Section $\mathrm{VI}$ we briefly discuss the arrow of time in physics and its relation to the time-arrow of feedback stability. Finally, in Section VII we consider feedback loops with small time delays and discuss the contrasting effects of delays and predictors and the connection with the arrow of time.

\section{TIME-ASYMMETRY AND STABILITY}

\section{A. Input-output and Lyapunov stability}

We focus on finite-dimensional linear dynamical systems which, for the most part, are assumed to be time-invariant. The dimensions of input, state and output (column) vectors, as well as the consistent sizes of transformation matrices in state-space models, are suppressed for notational simplicity. The following result is basic and well-known, cf. [38, p. 52-53], $[12$, p. 82].

Proposition 1: Let $\mathbf{P}$ be a linear time-invariant finite-dimensional system which is controllable and observable and is specified by

$$
\begin{aligned}
& \dot{x}=A x+B u, \\
& y=C x+D u,
\end{aligned}
$$

with an initial condition $x(0)=0$. Then $y \in \mathcal{L}_{2}[0, \infty)$ for all $u \in \mathcal{L}_{2}[0, \infty)$ if and only if the matrix $A$ is Hurwitz. Moreover, if this condition holds, $y$ is determined uniquely by $\hat{y}(s)=\left(C(s I-A)^{-1} B+D\right) \hat{u}(s)$, where ^ denotes the Laplace transform.

Many variants and extensions of the result are familiar: signal spaces with different norms can also be used; there is a finite-gain property relating the $\mathcal{L}_{2}$-norms of $y$ and $u$; even with $x(0) \neq 0$ the main equivalence in the proposition still holds. Here we would like to highlight the fact that the result establishes an equivalence between stability defined in terms of the forced response and stability defined in terms of the free response, i.e. an 
equivalence between bounded-input/bounded-output (BIBO) stability and Lyapunov stability for a system operating on the positive time-axis. Asymptotic stability in the sense of Lyapunov is obviously a time-asymmetric concept since convergence of the state vector is required as $t$ tends to PLUS infinity, starting from an arbitrary initial condition at $t=0$. In itself, BIBO stability does not appear to have this asymmetry, yet it is implicit in the formulation of Proposition 1 .

To further illustrate the point we can write down the following obvious corollary of Proposition 1, obtained by running time backwards from 0 to $-\infty$. By changing the support of the signal spaces from the positive half-line to the negative half-line stability defined through the forced response (BIBO stability) becomes equivalent to asymptotic stability in the sense of Lyapunov for the reversed time-direction as $t$ tends to MINUS infinity.

Proposition 2: Let $\mathbf{P}$ be a linear system as in Proposition 1 with $x(0)=0$. Then $y \in \mathcal{L}_{2}(-\infty, 0]$ for all $u \in \mathcal{L}_{2}(-\infty, 0]$ if and only if the matrix $-A$ is Hurwitz.

We now turn to the situation where inputs and outputs may have support on the doublyinfinite time-axis. In this case the following holds, e.g. see [44, p. 101].

Proposition 3: Let $\mathbf{P}$ be a linear system as in Proposition 1. Then there exists $y \in$ $\mathcal{L}_{2}(-\infty, \infty)$ for all $u \in \mathcal{L}_{2}(-\infty, \infty)$ if and only if $A$ has no imaginary-axis eigenvalues. Moreover, if this condition holds, $y$ is determined uniquely by $\hat{y}(s)=\left(C(s I-A)^{-1} B+\right.$ $D) \hat{u}(s)$.

We remark that Proposition 3 is the natural generalisation of Proposition 1 when systems are viewed as operators. A linear system in Proposition 1 becomes a multiplication operator on the Fourier transformed spaces. The operator is bounded if and only if the "symbol" (the transfer-function) belongs to $H_{\infty}$, which under the controllability and observability assumption is equivalent to $A$ being Hurwitz. On the double-axis a multiplication operator on the Fourier transformed spaces is bounded if and only if the symbol belongs to $L_{\infty}-$ which for rational symbols excludes only poles on the imaginary axis.

In Proposition 3 there is no longer any relationship between a notion of BIBO stability and Lyapunov stability (in either time-direction). Clearly, both $A$ and $-A$ may fail to be Hurwitz. Since only the existence of some $y \in \mathcal{L}_{2}(-\infty, \infty)$ is required for a given $u \in \mathcal{L}_{2}(-\infty, \infty)$, and the free motion solutions of (11) are ignored, this is not surprising. Propositions 1 and 2 , by contrast, establish a connection between BIBO stability and Lyapunov stability as $t \rightarrow+\infty$ (respectively, $t \rightarrow-\infty$ ) without putting in explicit requirements on the free motion solutions.

We now consider the feedback interconnection in the form of Fig. 1 where $\mathbf{P}$ and $\mathbf{C}$ are linear systems. The existence of signals $u_{i}, y_{j}(i, j \in\{1,2\})$ in $\mathcal{L}_{2}[0, \infty)$ which satisfy the feedback equations for a given pair of external inputs $u_{0}, y_{0}$ in $\mathcal{L}_{2}[0, \infty)$, for a given set of initial conditions, is a well-known and natural definition of stability in terms of the forced response. From Proposition 1 stability in this sense is equivalent to asymptotic stability in the sense of Lyapunov of the combined state-space (assuming minimal realizations for $\mathbf{P}$ and $\mathbf{C}$ and well-posedness). Again, BIBO stability inherits the required time-asymmetry from the asymmetry of the support interval $[0, \infty)$. 


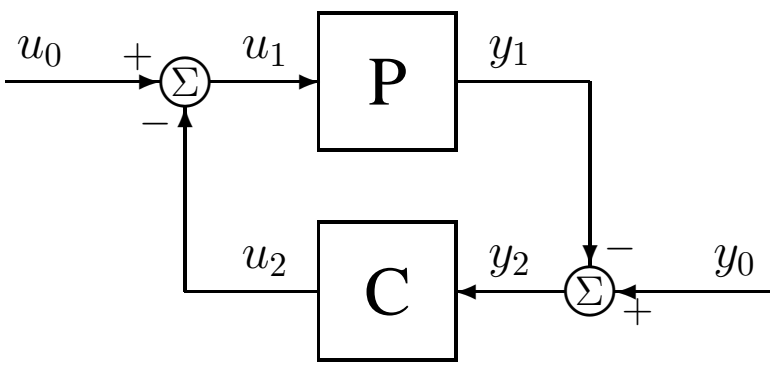

Fig. 1. Standard feedback configuration.

It is apparent that the corresponding definition of BIBO stability for this feedback interconnection with $\mathcal{L}_{2}(-\infty, \infty)$ signals, generalising Proposition 3 , will not correspond to a sensible notion of closed-loop stability. Indeed, we can easily check that a system $\mathbf{P}$ with transfer funtion $P(s)=1 /(s-1)$ is "stabilised" by any of the controllers with $C(s)=2$, $C(s)=0$, or $C(s)=-0.5 /(s+1)$. (In conventional terms the controllers give closed-loop poles which are in the open left-half plane (LHP), the open right-half plane (RHP), and in both half planes, respectively.)

We can summarize the points so far as follows. Stability is a time-asymmetric conceptthe requirement of an asymptotic property as $t$ tends to PLUS infinity defines a time arrow. If stability is defined by requiring bounded outputs in response to bounded inputs then a time arrow is not obviously implied. However, for signal spaces with support on a positive (resp. negative) half-line, the definition turns out to imply a positive (resp. negative) time arrow. On the other hand, a bounded-input bounded-output definition of stability for signals with support on the doubly-infinite time-axis does not define a preferred time arrow. Stable systems defined by bounded "multiplication operators" may be stable in the sense of Lyapunov in the positive time-direction, in the negative time-direction or in neither direction.

\section{B. The two-sided time axis and causality}

The fact that the doubly-infinite time axis causes problems for the analysis of stability and of stabilisation was pointed out in [8]. The explanation given there is consistent with that of Section $\amalg$, but the overall argument was somewhat different. We now summarize the reasoning of [8].

Two systems $\mathbf{P}_{i}(i=1,2)$ defined by convolution operators were considered:

$$
y(t)=\int_{-\infty}^{\infty} h_{i}(t-\tau) u(\tau) d \tau=h_{i} * u
$$

where $h_{1}(t)=e^{t}$ for $t \geq 0$ and zero otherwise, and $h_{2}(t)=-e^{t}$ for $t \leq 0$ and zero otherwise, respectively. Each system has (double-sided Laplace) transfer function equal to $1 /(s-1)$, but with differing regions of convergence. The first system is unstable and causal and the second is stable and non-causal (in fact anticausal) according to the usual definitions.

When viewed on $\mathcal{L}_{2}(-\infty, \infty), \mathbf{P}_{2}$ is a bounded operator and hence is a stable system in an input-output sense. On the other hand, it was shown in [8] that $\mathbf{P}_{1}$ fails to be stabilisable 
on $\mathcal{L}_{2}(-\infty, \infty)$. This is a counterintuitive result since $\mathbf{P}_{1}$ is stabilisable in the ordinary way on any positive half-line. The proof that $\mathbf{P}_{1}$ fails to be stabilisable on the doubly-infinite time-axis reduces to the observation that the graph of $\mathbf{P}_{1}$ fails to be closed.

It was also pointed out in [8] that the closure of the graph of $\mathbf{P}_{1}$ coincides with the graph of $\mathbf{P}_{2}$. Once the graph is closed there appears to be no problem with stabilisation. But in closing the graph "anti-causal" trajectories are brought in which are inconsistent with the convolution representation of the system, so this was considered inadmissible.

Another possible remedy discussed in [8] was to consider the underlying differential equation representations rather than the convolution representations. In fact both systems are defined by the same differential equation

$$
\dot{y}=y+u \text {. }
$$

More precisely, the trajectories of both $\mathbf{P}_{1}$ and $\mathbf{P}_{2}$ satisfy this equation. In terms of "flow of time" thinking, $\mathbf{P}_{1}$ appears to arise by solving this equation forwards in time while $\mathbf{P}_{2}$ is obtained by solving it backwards. This suggestion seems to make stronger the argument to consider $\mathbf{P}_{1}$ and $\mathbf{P}_{2}$ to be the same system. But this was considered unnatural in [8] on the grounds that it appears to abandon any notion of causality, or that it leaves the direction of time undefined.

The discussion of Section [II allows the difficulties pointed out in [8] to be explained in a new way. Let us suppose we are willing to accept the closure of the graph of $\mathbf{P}_{1}$ which makes it "stabilizable" on the double-axis in a bounded-input/bounded-output sense. As explained, $\mathbf{P}_{1}$ and $\mathbf{P}_{2}$ can now be thought of as one and the same system defined by (3) - a state-space description as in (1-2) solved forwards or backwards as desired. Does the closure of the graph resolve the difficulty pointed out in [8]? The answer is no, since the notion of stability does not correspond to the usual notions. As is made clear by Proposition 3. the feedback system may turn out to be stable in a conventional sense in the forward, backward or neither time-directions.

\section{The work of Mäkilä, Partington and Jacob}

A number of interesting observations and contributions have followed from [8] which we would like to comment on here.

The fact that a causal system on the double-axis can have a non-causal closure has led to a study of "closability" and "causal closability" as questions in their own right. Mäkilä [25] has shown that the lack of causal closability for the example of [8] extends to general $\mathcal{L}_{p}$ spaces on the double-axis. Jacob and Partington [17] give general characterisations of the graphs of time-invariant systems and derive necessary and sufficient conditions for the closure of a closable system to be causal. In [22] Mäkilä and Partington consider weighted $\mathcal{L}_{2}$-spaces on the double-axis and show that, when signals have very rapid decrease to zero towards $-\infty$, causal convolution operators may be closed operators. (So there is no issue of causality being lost due to the operation of closure.)

On the question of stabilization on the double time-axis, Jacob [15] has made an interesting suggestion. We have seen already that closing the graph and applying the BIBO 
stability definition fails to recover the usual concept of stability. Jacob proposed that causality of the closed-loop operators of the feedback system be added as an extra requirement. Jacob showed that the resulting characterisation of stability agrees with the usual definitions for linear time-invariant systems. In the context of the present paper we can re-interpret this result by saying that the causality condition forces the positive time-arrow into feedback system stability. We can understand this as follows. In [17] it is shown that a closed linear time-invariant system is causal on $\mathcal{L}_{2}(-\infty, \infty)$ if and only if the corresponding transfer function belongs to a certain Smirnov class. For finite dimensional systems this is equivalent to the transfer function having no right half-plane poles. Thus, in Proposition 3 , if $\mathbf{P}$ is required to be causal, BIBO stability agrees with Lyapunov stability with the positive timearrow.

Mäkilä and Partington in [22] make an interesting observation on the possible extension of Jacob's idea to the time-varying situation. They consider a causal, convolution operator derived from the underlying differential equation

$$
\dot{y}(t)+a(t) y(t)=u(t)
$$

where $a(t)=-1$ for $t \leq 0$ and $a(t)=+1$ for $t>0$ and point out that the closure of the $\mathcal{L}_{2}(-\infty, \infty)$ graph of the convolution system is not the graph of an operator. Essentially this boils down to the fact that there are free motion solutions $y(t)=c e^{-|t|}, u(t)=0$, where $c$ is a constant, which can be approximated arbitrarily closely by elements of the graph. This raises the question of whether the approach of Jacob can recover a theory of stabilization which is consistent with the single-axis case. At the same time it is pointed out that the system is stabilizable in a Lyapunov sense by the feedback

$$
u(t)=-2 y(t)
$$

In the present context this example highlights the care that is needed in defining stability for time-varying systems, even in the conventional sense. The open-loop system (4) is Lyapunov stable in the forward time-direction for any initial condition specified at any time (either positive or negative), but not uniformly so. Incidentally, the same is true for stability in the backwards time-direction. With the feedback (5) in force the system becomes uniformly stable in the sense of Lyapunov in the forward time-direction and unstable in reverse. In the perspective of the present paper, any method to force agreement between BIBO stability on the double-axis and conventional notions (such as requiring causality of the closed loop operators) might be seen as tantamount to directly imposing the desired time arrow within the stability definition.

In several papers (e.g., [24], [22], [23]) Mäkilä and Partington have advocated the use of a two-operator model for systems on the doubly infinite time-axis in the form $A y=B u$, where $A, B$ are causal, bounded operators, in contrast to a single-operator model $y=P u$, where $P$ is causal and possibly unbounded. Closed-loop stability is defined as the existence of a causal, bounded inverse of the feedback system operator mapping system inputs to exogenous disturbances. Since this definition incorporates a causality requirement on the closed-loop system there is evidently a close relationship between this idea and the approach of Jacob. 


\section{TIME-AsymmetRY AND Optimal REgUlATION}

This section focusses on the time-asymmetry of the definition of stability and its implications in the context of optimal regulation. Firstly, a time-conjugation operator will be defined as well as the concepts of f-stability and b-stability. Then the finite-horizon quadratic regulator problem will be considered for a system running forwards in time and backwards in time, and it will be shown that the optimal cost is generally different. The infinite-horizon (asymptotic) regulator will also be considered in the same way. It will be shown that the optimal cost can be expressed in terms of the two extremal solutions of the appropriate algebraic Riccati equation. The result shows that ease of optimal regulation depends on the time-direction.

\section{A. The time-conjugation operator, $f$-stability and b-stability}

Let $\mathbf{P}$ denote a dynamical system described by the state-space equations in (1-2), initialized at time zero and running forwards in time. Let $\mathfrak{J}$ denote the operation on $\mathbf{P}$ which corresponds to solving (1-2) backwards from $t=0$ followed by a flip of the time axis (so the new system runs forward again). More specifically we set $t_{1}=-t$, so that

$$
\frac{d}{d t}=-\frac{d}{d t_{1}}
$$

and then replace $t_{1}$ by $t$ which results in

$$
\begin{aligned}
-\dot{x} & =A x+B u, \text { with } x(0)=x_{0} \\
y & =C x+D u
\end{aligned}
$$

for the system $\mathfrak{J}(\mathbf{P})$. The effect on the transfer function is as follows: if $\mathbf{P}$ has transfer function $P(s)$, then $\mathfrak{J}(\mathbf{P})$ has transfer function $P(-s)$.

Define the system $\mathbf{P}$ to be $f$-stable if $A$ is Hurwitz, and define $\mathbf{P}$ to be $b$-stable if $\mathfrak{J}(\mathbf{P})$ is f-stable, or equivalently, if $-A$ is Hurwitz. It is immediately obvious that a linear time-invariant system of the type (1, 2) can never be both f-stable and b-stable. Similarly, a controller which makes (1,2) f-stable cannot make it b-stable as well.

\section{B. The finite-horizon linear quadratic regulator}

Let $\mathbf{P}$ be a linear time-invariant system which is controllable and observable and described by (1, 2), as before, with $D=0$ and $x(0)=x_{0}$. Consider the problem of the regulation of $\mathbf{P}$ with criterion

$$
J=\int_{0}^{T}\left(y(t)^{\prime} Q y(t)+u(t)^{\prime} R u(t)\right) d t+x(T)^{\prime} H x(T) .
$$

This has solution

$$
u(t)=-R^{-1} B^{\prime} S(t) x(t)
$$

where

$$
-\dot{S}(t)=S(t) A+A^{\prime} S(t)-S(t) B R^{-1} B^{\prime} S(t)+C^{\prime} Q C
$$


and $S(T)=H$, with optimal cost $J_{\mathrm{f}, \mathrm{T}}=x_{0}^{\prime} S(0) x_{0}$ [1]. When $\mathbf{P}$ runs backwards in time from $x(0)=x_{0}$ with cost

$$
J=\int_{-T}^{0}\left(y(t)^{\prime} Q y(t)+u(t)^{\prime} R u(t)\right) d t+x(-T)^{\prime} H x(-T)
$$

we can check that the optimal control is still given by (6) where $S(t)$ satisfies (7) with $S(-T)=-H$, and that the optimal cost is $J_{b, T}=-x_{0}^{\prime} S(0) x_{0}$. It can be readily verified that $S(0)$ (forward case) is in general different from $-S(0)$ (backward case), and so the optimal cost is different in the two cases, e.g., if $A=B=C=Q=R=T=1$ and $H=10$, then $S(0)=2.5415$ in the forward case and $-S(0)=0.5495$ in the backward case.

\section{The infinite-horizon linear-quadratic regulator}

Again let $\mathbf{P}$ be a linear time-invariant system which is controllable and observable and described by (1,2) with $D=0$ and $x(0)=x_{0}$. It is well-known [1] that

$$
J=\int_{0}^{\infty}\left(y(t)^{\prime} Q y(t)+u(t)^{\prime} R u(t)\right) d t
$$

has a minimum given by $J_{\mathrm{f}, \infty}=x_{0}^{\prime} S_{+} x_{0}$ where $S_{+}$is the unique positive-definite solution to the algebraic Riccati equation

$$
A^{\prime} S+S A-S B R^{-1} B^{\prime} S+C^{\prime} Q C=0 .
$$

It is also well-known that $S_{+}$is the unique solution of (9) for which $A-B R^{-1} B^{\prime} S$ has all its eigenvalues in the open LHP. In the language of the present paper we can say that $S_{+}$is the unique solution of (9) which makes the system (1, 2) f-stable with the controller $u=-R^{-1} B^{\prime} S x$.

What happens if we require the minimisation of

$$
J=\int_{-\infty}^{0}\left(y(t)^{\prime} Q y(t)+u(t)^{\prime} R u(t)\right) d t
$$

for (112) running backwards in time? This is the same as the conventional problem for the system $\mathfrak{J}(\mathbf{P})$. It is easy to see that the minimum is given by $J_{\mathrm{b}, \infty}=-x_{0}^{\prime} S_{-} x_{0}$ where $S_{-}$ is the unique negative-definite solution to (9). It is also well-known that $S_{-}$is the unique solution of (9) for which $A-B R^{-1} B^{\prime} S$ has all its eigenvalues in the open RHP [44]. In the language of the present paper we can say that $S_{-}$is the unique solution of (9) which makes the system (1-2) b-stable with the controller $u=-R^{-1} B^{\prime} S x$.

In general $J_{\mathrm{f}, \infty}=x_{0}^{\prime} S_{+} x_{0}$ and $J_{\mathrm{b}, \infty}=-x_{0}^{\prime} S_{-} x_{0}$ are different. This shows that "difficulty of control" is time-asymmetric for the standard linear-quadratic regulator on the infinite horizon. The difference can be significant, e.g. if $A=1, B=\epsilon, C=1, Q=1$ and $R=1$ then $S_{+}=2 / \epsilon^{2}+1 / 2+O\left(\epsilon^{2}\right)$ and $S_{-}=-1 / 2+O\left(\epsilon^{2}\right)$ for $\epsilon$ small. 


\section{Time-Asymmetry And Modelling UnCERTAinty}

In this section we look at the topology for uncertainty in feedback control and how this is affected by the time arrow. We will see that dynamical systems which are close in the usual sense, that a common controller can stabilise them and give a similar closed-loop behaviour, may not be close if time is reversed.

\section{A. The gap metric and robustness of stability}

Zames and El-Sakkary [43] introduced a metric on dynamical systems for the purpose of assessing robustness. This was based on the gap metric used in functional analysis to study invertibility of operators [19], [32]. Specifically, systems are considered to be operators on $\mathcal{L}_{2}[0, \infty)$ with a graph which is a closed subspace of $\mathcal{L}_{2}[0, \infty)$. Consider two linear systems $\mathbf{P}_{i}(i=1,2)$ with transfer functions

$$
P_{i}(s)=n_{i}(s)\left(m_{i}(s)\right)^{-1}
$$

where $n_{i}(s)$ and $m_{i}(s)$ are coprime polynomials or, more generally, right-coprime polynomial matrices. Let

$$
\left(n_{i}(-s)\right)^{T} n_{i}(s)+\left(m_{i}(-s)\right)^{T} m_{i}(s)=\left(d_{i}(-s)\right)^{T} d_{i}(s)
$$

with $\operatorname{det}\left(d_{i}(s)\right)$ a Hurwitz polynomial and ()$^{T}$ representing matrix transpose-the existence of such a polynomial (matrix) $d_{i}(s)$ is a standard result in the theory of canonical factorization [41]. Then,

$$
\mathcal{G}_{\mathbf{P}_{i}, \mathcal{H}_{2}}:=\left(\begin{array}{l}
m_{i}(s)\left(d_{i}(s)\right)^{-1} \\
n_{i}(s)\left(d_{i}(s)\right)^{-1}
\end{array}\right) \mathcal{H}_{2}:=G_{i}(s) \mathcal{H}_{2}
$$

is (the Fourier transform of) the graph of $\mathbf{P}_{i}$, for $i=1,2$. Thus, the graph symbol $G_{i}(s)$ generates the graph of $\mathbf{P}_{i}$ as its range. Then the gap between $\mathbf{P}_{1}$ and $\mathbf{P}_{2}$ is defined to be $\delta_{\mathcal{H}_{2}}\left(\mathbf{P}_{1}, \mathbf{P}_{2}\right):=\left\|\Pi_{\mathcal{G}_{\mathbf{P}_{1}, \mathcal{H}_{2}}}-\Pi_{\mathcal{G}_{\mathbf{P}_{2}, \mathcal{H}_{2}}}\right\|$ where $\Pi_{\mathcal{K}}$ denotes orthogonal projection onto a closed subspace $\mathcal{K}$.

Let the feedback configuration of Fig. 1 be denoted by $[\mathbf{P}, \mathbf{C}]$, where $\mathbf{P}$ and $\mathbf{C}$ are linear systems defined as operators on $\mathcal{L}_{2}[0, \infty)$ which may possibly be unbounded. Define

$$
\mathbf{H}_{\mathbf{P}, \mathbf{C}}:=\left(\begin{array}{l}
\mathbf{I} \\
\mathbf{P}
\end{array}\right)(\mathbf{I}-\mathbf{P C})^{-1}\left(\begin{array}{ll}
\mathbf{I} & -\mathbf{C}
\end{array}\right)
$$

to be the operator mapping $\left(\begin{array}{lll}u_{0}^{T} & y_{0}^{T}\end{array}\right)^{T}$ to $\left(\begin{array}{ll}u_{1}^{T} & y_{1}^{T}\end{array}\right)^{T}$. The following are basic robustness results for gap metric uncertainty.

Proposition 4: [9] Assume that the closed-loop system $[\mathbf{P}, \mathbf{C}]$ is f-stable. Then, $\left[\mathbf{P}_{1}, \mathbf{C}\right]$ is f-stable for all $\mathbf{P}_{1}$ such that $\delta_{\mathcal{H}_{2}}\left(\mathbf{P}, \mathbf{P}_{1}\right) \leq b$ if and only if $b<b_{\mathbf{P}, \mathbf{C}}$ where

$$
b_{\mathbf{P}, \mathbf{C}}:=\left\|\mathbf{H}_{\mathbf{P}, \mathbf{C}}\right\|_{\infty}^{-1} .
$$


Proposition 5: [43] Assume that the closed-loop system $[\mathbf{P}, \mathbf{C}]$ is f-stable. Then, the following are equivalent:

(i) $\delta_{\mathcal{H}_{2}}\left(\mathbf{P}_{n}, \mathbf{P}\right) \rightarrow 0$ as $n \rightarrow \infty$.

(ii) $\mathbf{H}_{\mathbf{P}_{n}, \mathbf{C}}$ is $\mathbf{f}$-stable for sufficiently large $n$ and $\left\|\mathbf{H}_{\mathbf{P}_{n}, \mathbf{C}}-\mathbf{H}_{\mathbf{P}, \mathbf{C}}\right\|_{\infty} \rightarrow 0$ as $n \rightarrow \infty$.

Proposition 5] was the primary justification for the claim in [43] that the gap metric defines the "correct" topology for robustness of feedback systems. In the present context, it can be seen that the choice of a signal space with support on the positive half-line is essential in achieving an appropriate topology. To emphasize the point, if $\mathcal{L}_{2}[0, \infty)$ were replaced by $\mathcal{L}_{2}(-\infty, 0]$ then the above proposition would hold with f-stability replaced by b-stability.

Let us consider the case where systems are defined on $\mathcal{L}_{2}(-\infty, \infty)$. Then we define

$$
\delta_{\mathcal{L}_{2}}\left(\mathbf{P}_{1}, \mathbf{P}_{2}\right):=\left\|\Pi_{\mathcal{G}_{\mathbf{P}_{1}, \mathcal{L}_{2}}}-\Pi_{\mathcal{G}_{\mathbf{P}_{2}, \mathcal{L}_{2}}}\right\|
$$

where

$$
\mathcal{G}_{\mathbf{P}_{i}, \mathcal{L}_{2}}:=G_{i}(s) \mathcal{L}_{2}
$$

and $\mathcal{L}_{2}:=\mathcal{L}_{2}(-j \infty, j \infty)$. With this definition, $\mathcal{G}_{\mathbf{P}, \mathcal{L}_{2}}$ is always closed, but may contain "non-causal" input-output pairs (as pointed out in [8]—see also Section [I-B). It is easy to construct examples to demonstrate that convergence of $\delta_{\mathcal{L}_{2}}\left(\mathbf{P}_{n}, \mathbf{P}\right)$ to zero does not allow any closed-loop stability prediction, e.g., $[\mathbf{P}, \mathbf{C}]$ f-stable does not imply $\left[\mathbf{P}_{n}, \mathbf{C}\right]$ f-stable for sufficiently large $n$.

In [36] Vinnicombe introduced a new metric $\delta_{v}(\cdot, \cdot)$ on dynamical systems which defines the same topology as $\delta_{\mathcal{H}_{2}}(\cdot, \cdot)$, and which satisfies the following inequality:

$$
\delta_{\mathcal{L}_{2}}(\cdot, \cdot) \leq \delta_{v}(\cdot, \cdot) \leq \delta_{\mathcal{H}_{2}}(\cdot, \cdot) .
$$

The v-gap between $\mathbf{P}_{1}$ and $\mathbf{P}_{2}$ is defined as follows:

$$
\delta_{v}\left(\mathbf{P}_{1}, \mathbf{P}_{2}\right):=\left\{\begin{array}{l}
\delta_{\mathcal{L}_{2}}\left(\mathbf{P}_{1}, \mathbf{P}_{2}\right) \text { if } \\
\text { wno }\left(\operatorname{det}\left(G_{2}(-s)^{T} G_{1}(s)\right)\right)=0, \\
1 \text { otherwise, }
\end{array}\right.
$$

where wno $(g(s))$ denotes the winding number about the origin of $g(s)$, as $s$ traces the standard Nyquist D-contour [36], [37]. A simple expression for $\delta_{\mathcal{L}_{2}}(\cdot, \cdot)$ can be obtained using left fractional representations-let $P_{i}(s)=\left(\tilde{m}_{i}(s)\right)^{-1} \tilde{n}_{i}(s)$ be a left-coprime polynomial fraction, $\tilde{d}_{i}$ the Hurwitz polynomial matrices which satisfy

$$
\tilde{n}_{i}(s)\left(\tilde{n}_{i}(-s)\right)^{T}+\tilde{m}_{i}(s)\left(\tilde{m}_{i}(-s)\right)^{T}=\tilde{d}_{i}(s)\left(\tilde{d}_{i}(-s)\right)^{T},
$$

and define

$$
\tilde{G}_{i}(s):=\left(-\left(\tilde{d}_{i}(s)\right)^{-1} \tilde{n}_{i}(s),\left(\tilde{d}_{i}(s)\right)^{-1} \tilde{m}_{i}(s)\right)
$$

for $i=1,2$. The graph of $\mathbf{P}_{i}$ is the kernel of multiplication by $\tilde{G}_{i}(s)$ (in the respective space of signals $\mathcal{H}_{2}$ or $\mathcal{L}_{2}$ ). The $\mathcal{L}_{2}$-gap can now be expressed as

$$
\delta_{\mathcal{L}_{2}}\left(\mathbf{P}_{1}, \mathbf{P}_{2}\right):=\left\|\tilde{G}_{2}(s) G_{1}(s)\right\|_{\infty} .
$$


It turns out that Propositions 4 and 5 both hold with $\delta_{\mathcal{H}_{2}}$ replaced by $\delta_{v}$ (see [36]). Since $\delta_{\mathcal{L}_{2}}=\delta_{v}$ when

$$
\operatorname{wno}\left(\operatorname{det}\left(G_{2}(-s)^{T} G_{1}(s)\right)\right)=0
$$

holds, this condition effectively imposes a positive time-arrow on the double-axis graph which forces f-stability to be retained under small perturbations in $\delta_{v}(\cdot, \cdot)$. This is illustrated by the following result (which can be readily derived from [36, Theorem 4.2]; see also [10]).

Proposition 6: Let $[\mathbf{P}, \mathbf{C}]$ be f-stable and suppose $\delta_{\mathcal{L}_{2}}\left(\mathbf{P}_{n}, \mathbf{P}\right) \rightarrow 0$ as $n \rightarrow \infty$. Then $\left[\mathbf{P}_{n}, \mathbf{C}\right]$ is f-stable for all sufficiently large $n$ if and only if $\operatorname{wno}\left(\operatorname{det}\left(G_{n}(-s)^{T} G(s)\right)\right)=0$ for all sufficiently large $n$.

\section{B. The effect of the time-arrow on gap distances}

We define a forward and a backward v-gap as follows,

$$
\begin{aligned}
\delta_{v, f}\left(\mathbf{P}_{1}, \mathbf{P}_{2}\right) & :=\delta_{v}\left(\mathbf{P}_{1}, \mathbf{P}_{2}\right) \\
\delta_{v, b}\left(\mathbf{P}_{1}, \mathbf{P}_{2}\right) & :=\delta_{v}\left(\mathfrak{J}\left(\mathbf{P}_{1}\right), \mathfrak{J}\left(\mathbf{P}_{2}\right)\right) .
\end{aligned}
$$

It is straightforward to see that

$$
\delta_{\mathcal{L}_{2}}\left(\mathbf{P}_{1}, \mathbf{P}_{2}\right)=\delta_{\mathcal{L}_{2}}\left(\mathfrak{J}\left(\mathbf{P}_{1}\right), \mathfrak{J}\left(\mathbf{P}_{2}\right)\right)
$$

so any difference between $\delta_{v, f}\left(\mathbf{P}_{1}, \mathbf{P}_{2}\right)$ and $\delta_{v, b}\left(\mathbf{P}_{1}, \mathbf{P}_{2}\right)$ lies in the winding number condition in (11). Let us examine this more closely. Note that

$$
\operatorname{det}\left(G_{2}(-s)^{T} G_{1}(s)\right)=\frac{h(s)}{\operatorname{det}\left(d_{2}(-s)\right) \operatorname{det}\left(d_{1}(s)\right)}
$$

where

$$
h(s):=\operatorname{det}\left(m_{2}(-s)^{T} m_{1}(s)+n_{2}(-s)^{T} n_{1}(s)\right) .
$$

If $\delta_{\mathcal{L}_{2}}\left(\mathbf{P}_{1}, \mathbf{P}_{2}\right)<1$ then it can be shown that wno $\left(\operatorname{det}\left(G_{2}(-s)^{T} G_{1}(s)\right)\right)$ is well-defined [36], in which case $h(s)$ admits a canonical factorization

$$
h(s)=h_{+}(s) h_{-}(s)
$$

where $h_{+}(s)$ and $h_{-}(-s)$ are Hurwitz polynomials. Thus, wno $\left(\operatorname{det}\left(G_{2}(-s)^{T} G_{1}(s)\right)\right)=0$ if and only if

$$
\operatorname{deg}\left(h_{+}(s)\right)=\operatorname{deg}\left(\operatorname{det}\left(d_{1}(s)\right)\right)
$$

or equivalently

$$
\operatorname{deg}\left(h_{-}(s)\right)=\operatorname{deg}\left(\operatorname{det}\left(d_{2}(s)\right)\right)
$$

It can be shown that the degree of $\operatorname{det}\left(\hat{d}_{i}(s)\right)$ is equal to the McMillan degree of $\mathbf{P}_{i}$ (e.g. using the uniqueness of normalised coprime factors over $H_{\infty}$ up to a constant unitary transformation and the corresponding state-space realisations [26], [35], [44]). Determining the graph symbol for $\mathfrak{J}\left(\mathbf{P}_{i}\right)$ requires a canonical factorization

$$
\left(n_{i}(s)\right)^{T} n_{i}(-s)+\left(m_{i}(s)\right)^{T} m_{i}(-s)=\left(\hat{d}_{i}(-s)\right)^{T} \hat{d}_{i}(s)
$$


with $\operatorname{det}\left(\hat{d}_{i}(s)\right)$ a Hurwitz polynomial. Again it can be shown that the $\operatorname{degree}$ of $\operatorname{det}\left(\hat{d}_{i}(s)\right)$ is equal to the McMillan degree of $\mathbf{P}_{i}$. The corresponding winding number condition in $\delta_{v, b}\left(\mathbf{P}_{1}, \mathbf{P}_{2}\right)$ can now be expressed as

$$
\operatorname{wno}\left(\operatorname{det}\left(\left(\hat{d}_{2}(-s)^{-1}\right) h(-s)\left(\hat{d}_{1}(s)^{-1}\right)\right)\right)=0
$$

which is equivalent to $\operatorname{deg}\left(h_{-}(s)\right)$ being equal to the McMillan degree of $\mathbf{P}_{1}$. We therefore obtain the following result.

Proposition 7: Let $P_{i}(s)(i=1,2)$ be the rational transfer functions of linear timeinvariant dynamical systems as above, with McMillan degrees $\mu_{i}$, and with $h, h_{+}, h_{-}$ as in (13, 14). Assume that $\delta_{\mathcal{L}_{2}}\left(\mathbf{P}_{1}, \mathbf{P}_{2}\right)<1$.

1) The following are equivalent:
a) $\delta_{v, f}\left(\mathbf{P}_{1}, \mathbf{P}_{2}\right)<1$,
b) $\operatorname{deg}\left(h_{+}(s)\right)=\mu_{1}$,
c) $\operatorname{deg}\left(h_{-}(s)\right)=\mu_{2}$.

2) The following are equivalent:
a) $\delta_{v, b}\left(\mathbf{P}_{1}, \mathbf{P}_{2}\right)<1$,
b) $\operatorname{deg}\left(h_{-}(s)\right)=\mu_{1}$,
c) $\operatorname{deg}\left(h_{+}(s)\right)=\mu_{2}$.

3) The following are equivalent:

a) $\delta_{v, f}\left(\mathbf{P}_{1}, \mathbf{P}_{2}\right)=\delta_{v, b}\left(\mathbf{P}_{1}, \mathbf{P}_{2}\right)<1$,

b) $\mu_{1}=\mu_{2}=\operatorname{deg}\left(h_{+}(s)\right)=\operatorname{deg}\left(h_{-}(s)\right)$.

In the above proposition, 1) expresses the zero winding number condition in (11) in an equivalent form, while 2) does the same for $\delta_{v, b}\left(\mathbf{P}_{1}, \mathbf{P}_{2}\right)$. It is interesting that when the two conditions are combined as in 3 ) the result is a very stringent requirement which includes the necessity that $P_{1}(s)$ and $P_{2}(s)$ have the same McMillan degree. This serves to highlight the fact that "unmodelled dynamics" which may account for a small error in $\delta_{v, f}$ (and which may be neglected in the design of a robust controller) will inevitably account for a substantial error in $\delta_{v, b}$.

Example 8: Consider two systems with different McMillan degrees, e.g. $P_{1}(s)=1$, $P_{2}(s)=1 / s$. It can be computed that $\delta_{v, f}\left(\mathbf{P}_{1}, \mathbf{P}_{2}\right)=1 / \sqrt{2}$. Proposition 7 then tells us immediately that $\delta_{v, b}\left(\mathbf{P}_{1}, \mathbf{P}_{3}\right)=1$ where $P_{3}(s)=-1 / s$. Similarly, if $P_{4}(s)=1 / s^{2}$, then Proposition 7 tells us that both $\delta_{v, f}\left(\mathbf{P}_{1}, \mathbf{P}_{4}\right)=\delta_{v, b}\left(\mathbf{P}_{1}, \mathbf{P}_{4}\right)=1$ since $P_{4}(s)=P_{4}(-s)$.

\section{TIME-ASYMMETRY AND ROBUST CONTROL}

This section addresses the implications of the time-asymmetry in the theory of robust control. In particular, we will also see that a system which is "easy" to control in one direction of time may be far from easy to control in the opposite direction. 


\section{A. Optimal robustness and difficulty of control}

In [11] it was shown that $b_{\mathbf{P}, \mathbf{C}}$ could be maximised over all stabilising $\mathbf{C}$ and that this amounts to solving a Nehari problem [44]. This optimum value, which we denote by

$$
b_{\text {opt }, \mathrm{f}}(\mathbf{P}),
$$

can be interpreted as a measure of ease/difficulty of control, where a value near to 1 means the plant is "easy to control" and a value near 0 means the plant is "hard to control".

With the understanding that $b_{\mathrm{opt}, \mathrm{f}}(\mathbf{P})$ has the meaning of "ease of control" with respect to the forward time-arrow for stabilty, it is interesting to define

$$
b_{\text {opt }, \mathrm{b}}(\mathbf{P}):=b_{\mathrm{opt}, \mathrm{f}}(\mathfrak{J}(\mathbf{P})),
$$

which represents "ease of control" with respect to the backwards time-arrow. Our main purpose in defining $b_{\text {opt,b }}(\mathbf{P})$ is to highlight the influence of the time-arrow in feedback regulation.

Let $\mathbf{P}$ be a controllable and observable system which is described by the state-space equations in (1-2), as before. Then, following [11], [9],

$$
b_{\mathrm{opt}, \mathrm{f}}(\mathbf{P})=\sqrt{1-\lambda_{\max }\left(Y_{+} X_{+}\right)}
$$

where $Y_{+}$is the positive definite solution of the Riccati equation

$$
\begin{aligned}
A_{0} Y+Y A_{0}^{*}-Y C R^{-1} C^{*} Y & \\
& +B\left(I-D^{*} R^{-1} D^{*}\right) B^{*}=0
\end{aligned}
$$

where $A_{0}=A-B D^{*} R^{-1} C$ and $R=I+D D^{*}$, and $X_{+}$is the corresponding solution to the $(Y$-dependent) Lyapunov equation

$$
\begin{array}{r}
\left(A_{0}-Y C^{*} R^{-1} C\right)^{*} X+X\left(A_{0}-Y C^{*} R^{-1} C\right) \\
+C^{*} R^{-1} C=0
\end{array}
$$

for $Y=Y_{+}$. Similarly, it can be seen that

$$
b_{\text {opt,b }}(\mathbf{P})=\sqrt{1-\lambda_{\max }\left(Y_{-} X_{-}\right)}
$$

where $Y_{-}$is the negative definite solution of the Riccati equation (15) while $X_{-}$is the corresponding solution to (16) for $Y=Y_{-}$.

In the following two examples we will see situations where $b_{\mathrm{opt}, \mathrm{f}}(\cdot)$ and $b_{\mathrm{opt}, \mathrm{b}}(\cdot)$ are very different.

Example 9: Near pole-zero cancellations. gives

Consider $P(s)=1+\frac{\epsilon}{s+1}$. Letting $-A=C=D=1$ and $B=\epsilon$ in equations (15)-(17)

$$
b_{\mathrm{opt}, \mathrm{f}}(\mathbf{P})=\sqrt{1-\frac{\sqrt{1+\epsilon+\epsilon^{2} / 2}-1-\epsilon / 2}{2 \sqrt{1+\epsilon+\epsilon^{2} / 2}}} .
$$


It follows that for small values of $\epsilon$,

$$
b_{\mathrm{opt}, \mathrm{f}}(\mathbf{P})=1-\frac{1}{32} \epsilon^{2}+O\left(\epsilon^{3}\right)
$$

and hence, $b_{\mathrm{opt}, \mathrm{f}}(\mathbf{P}) \rightarrow 1$ as $\epsilon \rightarrow 0$. On the other hand,

$$
b_{\mathrm{opt}, \mathrm{b}}(\mathbf{P})=\sqrt{1-\frac{\sqrt{1+\epsilon+\epsilon^{2} / 2}+1+\epsilon / 2}{2 \sqrt{1+\epsilon+\epsilon^{2} / 2}}} .
$$

which leads to

$$
b_{\mathrm{opt}, \mathrm{b}}(\mathbf{P})=\frac{1}{4}|\epsilon|+O\left(\epsilon^{2}\right)
$$

for small values of $\epsilon$, and hence $b_{\mathrm{opt}, \mathrm{b}}(\mathbf{P}) \rightarrow 0$ as $\epsilon \rightarrow 0$. This is accounted for by the fact that $P(s)$ has a near pole-zero cancellation in the LHP, which is innocuous for f-stabilisation, but highly challenging for b-stabilisation. The latter is equivalent to f-stabilisation of $P(-s)$, which has a troublesome near pole-zero cancellation in the RHP.

Example 10: Riding Bicycles.

A feedback stability problem in everyday experience is bicycle riding. An elementary model to study rider-bicycle stability is given in [2] which gives the following transfer function from steering angle input to tilt angle:

$$
\alpha V \frac{s+\beta V}{s^{2}-\gamma}
$$

where $\alpha, \beta, \gamma$ are positive constants and $V$ is the forward speed. This model has one RHP pole, but the zero is in the LHP. As such, this plant is not too difficult to control.

Let us consider what happens if we try to ride the bicycle backwards in time. This corresponds to trying to stabilise the plant $P(-s)$ forwards in time. The model still has one RHP pole, but the zero is also in the RHP, which makes stabilisation much more difficult. Indeed if $V \beta=\sqrt{\gamma}$ the plant is technically not stabilisable. It is interesting to note that an experimental bicycle with the steered wheel at the rear instead of the front has a transfer function from steering angle input to tilt angle given by [2] (see also [21])

$$
\alpha V \frac{-s+\beta V}{s^{2}-\gamma} .
$$

This is exactly the transfer function for the conventional bicycle ridden backwards in time.

Figure 2 shows the value of $b_{\mathrm{opt}, \mathrm{f}}$ and $b_{\mathrm{opt}, \mathrm{b}}$ versus $V$ with parameter values $\alpha=1 / 3$, $\beta=2$ and $\gamma=9$ (which are deemed reasonably realistic). Recall that $b_{\mathrm{opt}, \mathrm{b}}$ is the same as $b_{\text {opt,f }}$ for the rear-wheel steered bicycle model (19) at the same $V$. It can be observed that $b_{\text {opt,b }}$ is less than $b_{\text {opt,f }}$ for any $V$. Also, $b_{\text {opt,b }}$ is very small for low $V$, indicating difficulty of control, and zero at $V=1.5 \mathrm{~m} / \mathrm{s}$. For larger $V, b_{\mathrm{opt}, \mathrm{b}}$ increases, indicating that control becomes easier. These results are equivalent to the rear-wheeled steered bicycle being more difficult to ride than the front-wheel steered one, but still being reasonably controllable at higher speeds [2]. 


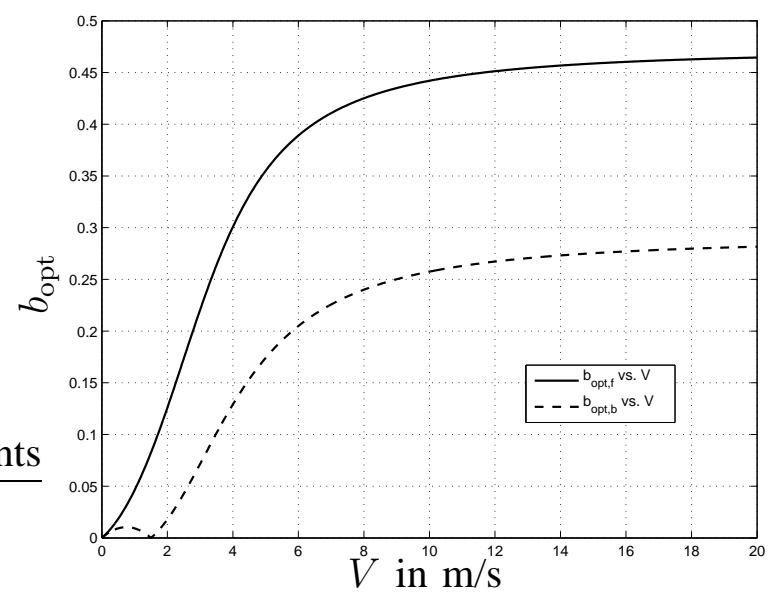

Fig. 2. $\quad b_{\mathrm{opt}, \mathrm{f}}$ and $b_{\mathrm{opt}, \mathrm{b}}$ versus $V$ for the bicycle model of (18) with $\alpha=1 / 3, \beta=2$, and $\gamma=9$.

\section{B. Time irreversible feedback phenomena}

The concept of ease or difficulty of control gives a thought-provoking perspective on reversibility. Systems which in a limiting situation are very difficult to control (in the sense that $b_{\mathrm{opt}, \mathrm{f}}(\mathbf{P})$ tends to zero) are unlikely to be observed in nature or technology. Nevertheless, such a system may be easy to control in the time-reversed direction (see Examples 9 and 10). This is independent of the fact that the underlying differential equation can be integrated equally well in either time-direction. This is reminiscent of phenomena (such as a bottle falling from the table and shattering into many pieces) that appear to be associated with an intrinsic direction of time even though classical physics would also allow the reversed motion as a solution (see Section VI for a further discussion).

We expand this point in the context of Example 10, The loss of stabilizability of the rearwheeled steered bicycle at $V=\sqrt{\gamma / \beta}$ has the following interesting consequence. Imagine a video of a rear-wheeled steered bicycle being ridden stably at this critical speed. Let us assume that it is possible to verify from the video the actual speed (e.g., by knowing the frame-rate and observing markings on the ground). An observer with a good grounding in control theory would be led to the inescapable conclusion that the video had been made when the said bicycle was actually being ridden backwards in space (i.e., with a negative $V$ ) and then played backwards in time as well, giving the impression of a forward motion.

\section{THE ARROW OF TIME IN PHYSICS}

The subject of the "arrow of time" is a well-known conundrum in physics. The second law of thermodynamics states that the entropy of a system increases with time. It is the time-asymmetry in this law which gives rise to the notion of the "thermodynamic arrow of time". The classical derivation of the second law in statistical mechanics due to Boltzmann is connected with a famous puzzle known as Loschmidt's paradox [40]. This essentially points out that the laws of mechanics used in the derivation of the second law are time-symmetric whereas the conclusion is not. Evidently the time-asymmetry creeps in through the statistical 
assumptions. An illuminating discussion of this issue is given in [29]. Other arrows of time have also been defined, for example (i) the "psychological arrow" - the direction in which time passes as perceived by a sentient being [14], [33], (ii) the "cosmological arrow"the direction of time in which the universe is expanding. Hawking [14] argues that the thermodynamic and psychological arrows are always aligned with each other but these need not always be aligned with the cosmological arrow (though they are at present).

In this paper we have described the time-asymmetry in the definition of control systems stability as a time-arrow. In the theory of dynamical systems there is also the notion of passivity, which again defines a time-arrow. For electrical circuits the time-arrow of passivity can be seen in the behaviour of the resistor, in contrast to the inductor and capacitor which are time-symmetric in their operation. If the electrical resistor were to operate backwards in time one would observe a resistor gathering low-grade heat from the environment and charging up a battery. This behaviour would be recognised as a violation of the second law of thermodynamics (see [20, pages 260, 390-2]). In a similar way, an ideal linear damper operating backwards in time extracts low-grade heat from the environment to create mechanical work, in violation of the second law. It seems that the arrow of time in passive systems or circuits coincides with, or is the same as, the thermodynamic arrow.

How does the arrow of time for control system stability relate to other time arrows? It is highly unlikely that a control engineer who is designing a control system for a plant will give even a moment's thought to the preferred time arrow for control. Without expressing the thought, the designer will seek decaying free motion solutions in the direction in which time is perceived to be passing. In this way the arrow of time for control could be said to coincide with the psychological arrow. On the other hand, in biological systems, active control is ubiquitous. It is less obvious that, for example, homeostasis in a cell is aligned with the psychological arrow. Here we will be content to raise the question of whether the stability arrow for control systems in general can be directly related to the thermodynamic arrow, e.g. by considering information flow or the effect of internal energy sources.

Finally, from a purely mathematical point of view, we observe that the arrow of time for control systems stability appears identical with the arrow of time for passivity. This supports the conclusion that the arrow of time for control systems stability always coincides with the thermodynamic (and psychological) arrow.

\section{FEEDBACK LOOPS AND TIME DELAYS}

Let $\mathbf{D}_{\tau}: x(t) \mapsto x(t-\tau)$ denote a time delay operator. It seems superfluous to say that $\mathbf{D}_{\tau}$ is physically realisable for $\tau>0$. Indeed the delay is a common feature of communication and control systems. For $\tau<0, \mathbf{D}_{\tau}$ is the ideal predictor which is not believed to be physically realisable as a "real-time" device. At first sight this "fact" appears to be self-evident, but its subtlety is revealed on closer examination-indeed, a rigorous justification appears not to be available at present. An insightful discussion of the issue of "causation" and its connection with the arrow of time is given in Price [29, Chapter 6]. Price's suggestion that the asymmetry of causation "is a projection of our own temporal asymmetry as agents in the world" [29, page 264] is similar to the view expressed by Bertrand Russell: "The law of causality, I believe, like much that passes muster among philosophers, is a relic 
of a bygone age, surviving, like the monarchy, only because it is erroneously supposed to do no harm". This prevalence in physics and philosophy of an anthropocentric explanation of causation sits in opposition to the belief of the unrealizability of a "prediction machine" out of physical components and processes, and suggests that a deeper analysis of the question is needed.

In this paper we will not attempt to further debate the origin and explanation of causation. In the next section we will simply highlight the striking difference in behaviour of feedback loops with small delays versus predictors and confirm the difference using the forward-time gap metric.

\section{A. Feedback stability, delays and predictors}

Consider a feedback system which consists of an integrator in series with a time delay and negative unity feedback. The governing equation is

$$
\dot{x}(t)+x(t-\tau)=d(t)
$$

where $d(t)$ denotes an external disturbance. We set $d(t) \equiv 0$ and consider the totality of all free motion solutions of the system equations. If all solutions decay as $t \rightarrow+\infty$ we say the system is f-stable. This definition agrees with the one given in Section III-A for finite-dimensional systems.

For $\tau \geq 0$ we can verify that (20) is f-stable. Taking Laplace transforms in (20) gives

$$
\hat{x}(s)=\frac{1}{s+e^{-s \tau}} \hat{d}(s) .
$$

We can verify that all zeros of $s+e^{-s \tau}=0$ are in the LHP so the system is f-stable.

Now consider the case where $\tau<0$. Note that this corresponds to an integrator with a predictor in negative feedback, which we would not expect to be realizable in the forward time direction. In fact, $s+e^{-s \tau}$ has infinitely many zeros in the RHP for any $\tau<0$ and hence the system fails to be f-stable. It is evident that the system displays a discontinuity in the asymptotic (as $t \rightarrow \infty$ ) behaviour of the free motion at the point $\tau=0$.

Let us now consider the closeness of the systems involved using the v-gap metric. Let $\mathbf{P}$ denote the integrator and $\mathbf{P}_{\tau}$ denote the integrator in series with $\mathbf{D}_{\tau}$. Regarding these as operators on $\mathcal{L}_{2}[0, \infty)$ we have the graph:

$$
\mathcal{G}_{\mathbf{P}_{\tau}, \mathcal{H}_{2}}=\left(\begin{array}{c}
\frac{s}{s+1} \\
\frac{e^{-s \tau}}{s+1}
\end{array}\right) \mathcal{H}_{2}
$$

for $\tau \geq 0$. Then

$$
\delta_{\mathcal{L}_{2}}\left(\mathbf{P}, \mathbf{P}_{\tau}\right)=\left\|\frac{s}{(s+1)^{2}}\left(1-e^{-s \tau}\right)\right\|_{\infty}
$$

which tends to zero as $\tau \rightarrow 0$. Also

$$
G_{2}(-s)^{T} G_{1}(s)=\frac{-s^{2}+e^{s \tau}}{-s^{2}+1}
$$


so providing $|\tau|<\pi$ there are no crossings of the negative real axis of this function when $s=j \omega$. Hence, $\operatorname{wno}\left(G_{2}(-s)^{T} G_{1}(s)\right)=0$, for $\tau$ sufficiently small. This implies

$$
\delta_{v, f}\left(\mathbf{P}, \mathbf{P}_{\tau}\right)=\delta_{\mathcal{L}_{2}}\left(\mathbf{P}, \mathbf{P}_{\tau}\right)
$$

for $\tau \geq 0$ and sufficiently small and $\delta_{v, f}\left(\mathbf{P}, \mathbf{P}_{\tau}\right) \rightarrow 0$ as $\tau \rightarrow 0$.

Now consider the case of $\mathbf{P}_{\tau}$ with $\tau<0$. Again regarding $\mathbf{P}_{\tau}$ as an operator on $\mathcal{L}_{2}[0, \infty)$ we have:

$$
\mathcal{G}_{\mathbf{P}_{\tau}}=\left(\begin{array}{c}
\frac{s e^{s \tau}}{s+1} \\
\frac{1}{s+1}
\end{array}\right) \mathcal{H}_{2}
$$

and $\delta_{\mathcal{L}_{2}}\left(\mathbf{P}, \mathbf{P}_{\tau}\right)=\left\|\frac{s}{(s+1)^{2}}\left(e^{s \tau}-1\right)\right\|_{\infty}$ which tends to zero as $\tau \rightarrow 0$. Also,

$$
G_{2}(-s)^{T} G_{1}(s)=\frac{-s^{2} e^{-s \tau}+1}{-s^{2}+1},
$$

which behaves like $e^{-s \tau}$ for large $s$, so the winding number of this function is not zero and $\delta_{v, f}\left(\mathbf{P}, \mathbf{P}_{\tau}\right)=1$ for $\tau<0$.

The above analysis with the gap agrees with the earlier conclusion on f-stability. For $\tau \geq 0$, f-stability was retained for sufficiently small $\tau$, but lost for any $\tau<0$. Now we have seen that, as long as $\tau \geq 0$, there is a small error in $\delta_{v, f}$, but for any $\tau<0, \delta_{v, f}\left(\mathbf{P}, \mathbf{P}_{\tau}\right)=1$.

Finally, it is interesting to mention that the tolerance of feedback loops to small timedelays is guaranteed by a well-known sufficient condition that the high-frequency loop-gain of the feedback loop is smaller than one ([3], [6], [39], [42]) - a condition routinely met in practice. It is easy to check that robustness to an arbitrarily small "parasitic predictor" in the loop would be guaranteed theoretically by the loop-gain being greater than one at arbitrarily high frequencies - a condition that appears impossible to achieve in a real feedback system.

\section{SYNOPSIS}

1) Stability is a time-asymmetric concept. The requirement of an asymptotic property as $t$ tends to PLUS infinity defines a time arrow.

2) A stability definition which requires bounded outputs in response to bounded inputs does not obviously imply a time arrow. For signal spaces with support on a positive (resp. negative) half-line, the definition turns out to imply a positive (resp. negative) time arrow.

3) A bounded-input bounded-output definition of stability for signals with support on the doubly-infinite time-axis does not define a preferred time arrow. Stable systems defined by bounded multiplication operators may be stable in the sense of Lyapunov in the positive time direction, in the negative time direction or in neither direction.

4) The fact that the closure of the graph of an unstable causal system may coincide with the graph of a stable anti-causal system on the doubly-infinite time-axis need not be a fundamental obstacle in developing a usable control theory on the doubly-infinite time-axis.

5) Any method which modifies the BIBO definition of stability on the doubly-infinite time-axis to agree with conventional stability notions could be interpreted as the imposition of a positive time-arrow. 
6) A time-conjugation operator on systems was defined as well as the concepts of fstability and b-stability.

7) Both the finite-horizon and infinite-horizon quadratic regulators give a different optimal cost for a system running forwards in time and backwards in time. In the infinite horizon case the optimal cost can be expressed in terms of the two extremal solutions of the appropriate algebraic Riccati equation.

8) The role of the positive time arrow in the gap metric measure of uncertainty for dynamical systems was highlighted. The usual $\mathcal{H}_{2}$-gap metric inherits the positive time arrow by virtue of systems being defined as operators on the positive half-line. The $\mathcal{L}_{2}$-gap metric, which is well known to define an inappropriate topology for robust control, does not have a preferred time-direction due to the underlying operators being defined on the double-axis. The v-gap metric may be interpreted as the $\mathcal{L}_{2}$-gap with an imposed time-arrow.

9) A time-conjugated v-gap metric was defined to measure closeness for robust b-stabilisation. It was seen that closeness of systems in the forward and backwards directions is a strong condition which includes the requirement of equal McMillan degrees.

10) It was seen that ease or difficulty of control as measured by optimal robustness in the gap metric is a property that depends on the time-arrow.

11) The situation of a plant which is easy to control in one time-direction but impossible to control in the other shows that irreversibility can be intimately related to control.

12) An engineering perspective of control suggests a close link between the control system stability arrow and the psychological arrow. Unified mathematical frameworks for passive circuits and feedback control suggest a close link between the control system stability arrow and the thermodynamic arrow. The question was raised whether the stability arrow for control systems can be directly related to the thermodynamic arrow.

13) The issue of the non-realizability of the pure predictor as a "real-time" device and the connection with the arrow of time was highlighted as well as the difficulty of establishing non-realizability rigorously. The strongly contrasting behaviour of feedback loops in the presence of arbitrarily small time-delays or predictors was pointed out.

\section{ACKNOWLEDGEMENT}

We are grateful to Jan Willems for helpful comments on an earlier draft.

\section{REFERENCES}

[1] B.D.O. Anderson and J.B. Moore, Optimal control: linear quadratic methods, Prentice-Hall, 1990.

[2] K.J. Aström, R.E. Klein, and A. Lennartsson, "Bicycle dynamics and control: adapted bicycles for education and research," IEEE Control Systems Magazine, 25 (4): 26-47, August 2005.

[3] J.F. Barman, F.M. Callier, and C.A. Desoer, " $L^{2}$-stability and $L^{2}$-instability of linear time-invariant distributed feedback systems perturbed by a small delay in the loop," IEEE Trans. on Automatic Contr. , 18(5): 479-484, October 1973.

[4] R. W. Brockett and J. C. Willems, "Stochastic control and the second law of thermodynamics," in the Proc. of the IEEE Conference on Decision and Control, San Diego, California, pp. 1007-1011, 1978

[5] H. Sandberg, J.C. Delvenne, and J.C. Doyle, "Linear-quadratic-Gaussian heat engines," in the Proc. of the IEEE Conference on Decision and Control, pages 3102-3107, December 2007.

[6] T.T. Georgiou and M.C. Smith, "w-Stability of feedback systems," Systems \& Control Letters, 13 (4): 271-277, November 1989. 
[7] T.T. Georgiou and M.C. Smith, "Graphs, causality and stabilizability: linear, shift-invariant systems on $L_{2}[0, \infty)$," Math. of Control Signals and Systems, 6, 195-223, 1993.

[8] T.T. Georgiou and M.C. Smith, "Intrinsic difficulties in using the doubly-infinite time axis for input-output systems theory," IEEE Trans. on Automatic Contr. , 40(3): 516-518, March 1995.

[9] T.T. Georgiou and M.C. Smith, "Optimal robustness in the gap metric," IEEE Trans. on Automat. Control, 35, 673-686, 1990.

[10] T.T. Georgiou, C. Shankwitz and M.C. Smith, "Identification of linear systems: a stochastic approach based on the graph," Proceedings of the 1992 American Control Conference, Chicago, June 1992, pp. 307-312.

[11] K. Glover and D. McFarlane, "Robust stabilization of normalized coprime factor plant descriptions with $H_{\infty}$ bounded uncertainty," IEEE Trans. on Automat. Contr., vol. 34, pp. 821-830, 1989.

[12] M. Green and D.J.N. Limebeer, Linear Robust Control, Prentice Hall, 1995.

[13] W. M. Haddad, V. S. Chellaboina, and S. G. Nersesov, Thermodynamics: A Dynamical Systems Approach, Princeton University Press, 2005.

[14] S.W. Hawking, A brief history of time, Bantam Books, 1988.

[15] B. Jacob, "What is the better signal space for discrete-time systems: $\ell_{2}(\mathbb{Z})$ or $\ell_{2}\left(\mathbb{N}_{0}\right)$ ?" SIAM J. Contr. and Opt., 43 (4): 1521-1534, 2004.

[16] B. Jacob, "An operator theoretical approach towards systems over the signal space $\ell_{2}(\mathbb{Z})$ ", Integral Equations and Operator Theory, 46 (2): 189-214, June 2003.

[17] B. Jacob, J.R. Partington, "Graphs, closability, and causality of linear time-invariant discrete-time systems," International J. on Control, 73 (11): 1051-1060, July 2000.

[18] J. B. Johnson, "Thermal agitation of electricity in conductors," Phys. Rev., 32: 97-109, July 1928.

[19] M.G. Kreln and M.A. Krasnosel'skii, "Fundamental theorems concerning the extension of Hermitian operators and some of their applications to the theory of orthogonal polynomials and the moment problem (in Russian)," Uspekhi Mat. Nauk., vol. 2, pp. 60-106, 1947.

[20] D. Kondepudi and I. Prigogine, Modern thermodynamics: from heat engines to dissipative structures, John Wiley \& Sons, 1998.

[21] D.J.N. Limebeer and R.S. Sharp, "Bicycles, Motorcycles, and Models," IEEE Control Systems Magazine, 26 (5) 34-61, October 2006.

[22] P.M. Makila and J.R. Partington, "A two-operator approach to robust stabilization of linear systems on $\mathbb{R}$," International J. on Control, 79 (9): 1026-1038 Sept. 2006.

[23] P.M. Makila, J.R. Partington, "Input-output stabilization of linear systems on $\mathbb{Z}$, , IEEE Trans. on Automat. Control, 49 (11): 1916-1928, November 2004.

[24] P.M. Makila, J.R. Partington, "Input-output stabilization on the doubly-infinite time axis," International J. on Control, 75 (13): 981-987, September 2002.

[25] P.M. Makila, "When is a linear convolution system stabilizable?" Systems \& Control Letters, 46 (5): 371-378, August 2002.

[26] D.G. Meyer and G.F. Franklin, "A connection between normalized coprime factorizations and linear quadratic regulator theory", IEEE Trans. on Automat. Contr., 32, 227-228, 1987.

[27] S.K. Mitter and N.J. Newton, "Information and entropy flow in the Kalman-Bucy filter," J. of Statistical Physics, 118: 145-176, 2005.

[28] H. Nyquist, "Thermal agitation of electric charge in conductors," Phys. Rev., 32: 110-113, July 1928.

[29] H. Price, Time's Arrow and Archimedes' Point, Oxford University Press, New York, 1996.

[30] B. Russell, "On the Notion of Cause", Proceedings of the Aristotelian Society, 13 (1913), pp. 1-26.

[31] H. Sandberg, J.C. Delvenne, and J.C. Doyle, "The Statistical Mechanics of Fluctuation-Dissipation and Measurement Back Action," in the Proc. of the American Control Conference, 2007, available at http://arxiv.org/abs/math.DS/0611628

[32] B. Sz.-Nagy, "Perturbations des transformations autoadjointes dans l'espace de Hilbert," Comm. Math. Helv., vol. 19, pp. 347-366, 1947.

[33] L.S. Schulman, Time's arrow and quantum measurement, Cambridge University Press, 1997.

[34] B.W. Schumacher, "Demonic Heat Engines", in Physical Origins of Time Asymmetry, Eds. J.J. Halliwell, J. PérezMercader, and W.H. Zurek, Cambridge University Press, 1994.

[35] M. Vidyasagar, "Normalised coprime factorizations for nonstrictly proper systems", IEEE Trans. on Automat. Contr., 33, 300-301, 1988.

[36] G. Vinnicombe, "Frequency domain uncertainty and the graph topology," IEEE Trans. on Automat. Control, 38, 1371-1383, 1993.

[37] G. Vinnicombe, Uncertainty and Feedback: $H_{\infty}$ loop-shaping and the $\nu$-gap metric, Imperial College Press, 2001.

[38] J.L. Willems, Stability Theory of Dynamical Systems, Thomas Nelson and Sons Ltd., London, 1970.

[39] J. C. Willems, The Analysis of feedback systems, MIT Press, 1971.

[40] http://en.wikipedia.org/wiki/Loschmidt's_paradox

[41] D.C. Youla, "On the factorization of rational matrices," IRE Transactions of Information Theory, 7, 172-189, 1961. 
[42] G. Zames, "Realizability Condition for Nonlinear Feedback Systems," IEEE Trans. on Circuits Theory, 11(2): 186194, June 1964.

[43] G. Zames and A.K. El-Sakkary, "Unstable systems and feedback: The gap metric," Proceedings of the Allerton Conference, pp. 380-385, October 1980.

[44] K. Zhou, J.C. Doyle and K. Glover, Robust and optimal control, Prentice-Hall, 1995. 